Artículo

\title{
Antagonismo de Trichoderma harzianum contra la fusariosis del garbanzo y su efecto biofertilizante
}

\author{
Talina Olivia Martínez-Martínez ${ }^{1}$ \\ Brenda Zulema Guerrero-Aguilar ${ }^{1}$ \\ Víctor Pecina-Quintero ${ }^{1}$ \\ Patricia Rivas-Valencia ${ }^{2}$ \\ Enrique González-Pérez ${ }^{1}$ \\ Juan Gabriel Angeles-Núñez ${ }^{1 \S}$
}

${ }^{1}$ Campo Experimental Bajío-INIFAP. Carretera San Miguel de Allende-Celaya km 6.5, Celaya, Guanajuato, México. CP. 38110. Tel. 5538718700, ext. 85242. (martinez.talina@inifap.gob.mx; guerrero.brenda@inifap.gob.mx; pecina.victor@inifap.gob.mx; gonzalez.enrique@inifap.gob.mx. ${ }^{2}$ Campo Experimental Valle de México-INIFAP. Carretera Los Reyes-Texcoco km 13.5, Coatlinchán, Texcoco, México. CP. 56250. Tel. 5538718700, ext. 85214. (rivas.patricia@inifap.gob.mx).

${ }^{\S}$ Autor para correspondencia: angeles.gabriel@inifap.gob.mx.

\section{Resumen}

El garbanzo es una leguminosa, que se cultiva en dos regiones de México principalmente, noroeste (Sonora, Sinaloa y Baja california) y la región de El Bajío (Guanajuato, Michoacán y Jalisco); sin embargo, cada año la producción del cultivo está comprometida con la fusariosis vascular, una de las principales enfermedades que afectan al cultivo y que está asociada al complejo fúngico Fusarium oxysporum, Fusarium solani, Rhizoctonia solani, Macrophomina phaseolina y Sclerotium rolfsii. Una alternativa de control biológico es la aplicación de Trichoderma, la que además tiene un efecto indirecto en la nutrición de la planta. El objetivo de este estudio fue determinar el antagonismo in vitro de dos cepas de Trichoderma harzianum (T1 y T2) y su efecto como biofertilizante. Se realizaron confrontaciones in vitro contra cepas de las razas de Fusarium oxysporum f. sp. ciceris (Foc 0, 1B/C, 5 y 6), Fusarium solani, Macrophomina phaseolina (MSonora y M-GTO) y Sclerotium rolfsii. Se evaluó el efecto de T2 como biofertilizante (TB) midiendo las variables: número de flores, vainas, altura de la planta, diámetro del tallo, longitud de la raíz y rendimiento de grano. Las dos cepas de $T$. harzianum mostraron antagonismo en diferente escala contra los patógenos. Adicionalmente, con el tratamiento donde se aplicó T. harzianum (TB) se presentaron incrementos en el número de flores (30\%), vainas (24\%), altura (3\%), diámetro de las plantas (3.5\%), así como la longitud de la raíz (13\%) y rendimiento del grano (23\%).

Palabras claves: crecimiento de cultivo, micoparasitismo, rendimiento de grano.

Recibido: febrero de 2020

Aceptado: junio de 2020 


\section{Introducción}

Las enfermedades representan una de las causas en la disminución de los rendimientos en el cultivo de garbanzo, entre las más importantes en el mundo, se encuentran las ocasionadas por hongos del suelo, como son: Fusarium oxysporum Schltdl., Fusarium solani (Mart.) Sacc., Rhizoctonia solani Kühn, Macrophomina phaseolina (Tassi) Goid y Sclerotium rolfsii Sacc.; sin embargo, por los daños que producen y la frecuencia con que se presentan, Fusarium spp. Es de mayor importancia, especialmente la forma especial ciceris.

Esta enfermedad denominada fusariosis, marchitez o rabia del garbanzo aparece, principalmente, en los suelos arcillosos o con problemas de drenaje, condición que favorece el desarrollo de estas patologías (Jiménez-Gasco et al., 2004). Las pérdidas en el rendimiento cuantificadas con el peso de 100 semillas pueden ser de $82 \%$ y hasta de $100 \%$ en variedades susceptibles (Navas-Cortes $e t$ al., 2000). Fusarium oxysporum f. sp. ciceris (FOC) produce clamidosporas que pueden sobrevivir en el suelo en ausencia del hospedero por más de seis años (Haware et al., 1996).

Este patógeno se disemina rápidamente, ataca la raíz de la planta y causa un estrés hídrico y deficiencia de nutrientes debido a la oclusión de los vasos conductores del xilema, además de clorosis, necrosis y abscisión de hojas (Jiménez-Gasco et al., 2004). Se han descrito ocho razas de FOC: 0, 1A, 1B/C, 2, 3, 4, 5 y 6 (Haware y Nene, 1982; Jiménez-Díaz et al., 1993) y dos biotipos de patogenicidad: amarillamiento y marchitez.

En el primero se presenta amarillamiento foliar y coloración vascular, la planta muere a los 40 días de inoculación del patógeno. En el de marchitez se observa clorosis severa, flacidez y coloración vascular, la planta muere a los 20 días de la inoculación (Jiménez-Gasco et al., 2004). Las razas 0 y $1 \mathrm{~B} / \mathrm{C}$ corresponden a patotipos de amarillamiento, mientras que las razas $1 \mathrm{~A}, 2,3,4,5$ y 6 a patotipos de marchitez (Arvayo-Ortiz et al., 2011).

En tanto, Macrophomina phaseolina se presente comúnmente cuando existen temperaturas altas y baja humedad del suelo, debido a su capacidad de formar esclerocios permite que el hongo sobreviva a condiciones ambientales adversas, puede ocasionar pérdidas debido a la disminución en la longitud de la raíz (44-49\%), longitud de los brotes (5-16\%) y peso fresco (55-63\%) (Khan et al., 2017). Sclerotium rolfsii puede provocar una mortalidad de 55-95\% de las plántulas de garbanzo, altas precipitaciones y temperaturas superiores a los $25^{\circ} \mathrm{C}$ son condiciones óptimas para causar enfermedad, sobrevive en forma de micelio en los restos de plantas y como estructuras escleróticas en suelo (Sharma y Ghosh, 2017).

Debido a lo anterior, se requiere de la identificación de agentes de biocontrol para contrarrestar los efectos de estos patógenos, alternativos al control convencional con productos químicos, lo cual representa un riesgo severo para la salud humana y contribuye al aumento de la contaminación al medio ambiente (Abdel-Monaim et al., 2011), además de que han dado lugar a la aparición de microorganismos altamente resistentes que conducen a enfermedades fúngicas con mayor incidencia (Hoyos-Carvajal et al., 2019).

Una alternativa es la utilización de hongos del género de Trichoderma, al que se le reconoce como agente de biocontrol debido a su capacidad de antibiosis, micoparasitismo, competencia por espacio y nutrientes, así como la producción de metabolitos secundarios (Hernández-Melchor et 
al., 2019). La mayoría de las especies de Trichoderma poseen un crecimiento y desarrollo acelerado, pueden tolerar condiciones ambientales extremas y son capaces de parasitar, controlar y destruir hongos, nemátodos y otros fitopatógenos (Ruiz Cisneros et al., 2018), además de tolerar la presencia de agroquímicos.

En garbanzo Trichoderma se ha destacatado por su capacidad antagónica (Rajput et al., 2010) y el control de patógenos como Rhizoctonia solani, Fusarium solani, Sclerotinia sclerotiorum, Fusarium oxysporum f. sp. ciceri (Abdel-Monaim et al., 2011; Khan et al., 2014). Diferentes investigaciones demuestran que el uso de control biológico es una alternativa viable para el control de la fusariosis en garbanzo, además de que mejora las características microbiológicas del suelo y por ende sus características fisicoquímicas, al mismo tiempo promueve el crecimiento a través de la producción de hormonas de crecimiento, solubilización de minerales y la inducción de resistencia sistémica (Hernández-Melchor et al., 2019).

Dada la información que se ha desarrollado por diversas investigaciones, se ha considerado la posibilidad de emplear cepas de Trichoderma para el control de la fusariosis y su posible efecto en el desarrollo del cultivo de garbanzo. Bajo este contexto, se plantearon los siguientes objetivos: 1) determinar el efecto antagónico in vitro de dos cepas de $T$. harzianum (T1 y T2) para el control biológico de hongos fitopatógenos causantes de la fusariosis del garbanzo; 2) evaluar el efecto in vivo de la aplicación de T2 sobre el desarrollo del cultivo de garbanzo.

\section{Materiales y métodos}

\section{Material biológico}

Se emplearon cuatro aislados de la raza de Fusarium oxysporum f. sp. ciceris (Foc). La raza Foc 5 provenientes de la zona Noroeste y las razas Foc. 0, Foc. 1 B/C y Foc 6 de la zona de El Bajío Guanajuatense, las cepas se identificaron previamente por medio marcadores moleculares tipo SCARs y RAPDs descritos por Jiménez-Gasco et al. (2004). Una cepa de F. solani y Sclerotium rolfsii, y dos cepas de Macrophomina phaseolina aisladas de Sonora (M-Sonora) y Guanajuato (M-GTO).

Las cepas de Trichoderma harzianum (T1 y T2) fueron donadas por el Campo Experimental Bajío (CEBAJ) del Instituto Nacional de Investigaciones Forestales, Agrícolas y Pecuarias (INIFAP), aisladas de suelos agrícolas de Valle de Santiago, Guanajuato. Se empleó semilla de garbanzo tipo blanco var. Blanoro (cosecha primavera otoño 2013) proporcionada por el banco de germoplasma del CEBAJ, cuyo resguardo fue a $-4{ }^{\circ} \mathrm{C}$ con $80 \% \mathrm{HR}$.

\section{Pruebas de antagonismo in vitro (cultivos duales)}

Se evaluaron los mecanismos de acción antagónica de T1 y T2 frente los ocho fitopatógenos involucradas en la fusariosis del garbanzo (Foc 250. Foc. 71, Foc 27, Foc 12, Macrophomina phaseolina y Sclerotium rolfsii) empleando la técnica de cultivos duales (Dennis and Webster, 1971). La evaluación se realizó mediante la observación de los mecanismos de competencia, micoparasitismo y antibiosis de acuerdo con la escala descrita por Bell et al. (1982) (Cuadro 1). 
Cuadro 1. Capacidad antagónica de Trichoderma según la escala del Bell et al. (1982).

\begin{tabular}{cl}
\hline Clase & Capacidad antagónica \\
\hline 1 & $\begin{array}{l}\text { Trichoderma coloniza completamente al fitopatógeno y cubre totalmente la superficie } \\
\text { del medio }\end{array}$ \\
2 & $\begin{array}{l}\text { Trichoderma coloniza las dos terceras partes de medio de cultivo, limitando el } \\
\text { crecimiento del fitopatógeno } \\
\text { Trichoderma y el patógeno colonizan cada uno la mitad de la superficie, el crecimiento } \\
\text { es similar }\end{array}$ \\
4 & $\begin{array}{l}\text { El fitopatógeno coloniza las dos terceras partes de la superficie del medio de cultivo y } \\
\text { limita el crecimiento de Trichoderma } \\
\text { El fitopatógeno coloniza completamente el medio del cultivo y crece sobre } \\
\text { Trichoderma }\end{array}$ \\
\end{tabular}

Los discos se colocaron en los extremos equidistantes de la caja y se incubaron a temperatura ambiente $\left(22^{\circ} \mathrm{C}\right)$. La evaluación de la capacidad antagónica se calculó estimando el porcentaje de inhibición del crecimiento radial (PICR). Este se obtuvo a partir del crecimiento de cada patógeno en cultivo dual, con respecto a los testigos, empleando la fórmula utilizada por Suárez et al. (2008).

Las pruebas se realizaron con cinco repeticiones (placas), en un diseño factorial de dos factores (cepa de Trichoderma y fitopatógenos), se realizó un análisis de varianza y comparación de medias Tukey $(p \leq 0.05)$. El nivel de micoparasitismo se estimó considerando los criterios establecidos por Widyastuti (2006), para las observaciones microscópicas se utilizó la técnica de impronta con diurex, se empleó un microscopio compuesto Carl Zeiss Axiostar plus con un campo de visión de $40 X$.

\section{Efecto de Trichoderma harzianum en el cultivo de garbanzo}

Se estableció una parcela de garbanzo de la variedad Blanoro con un diseñó de bloques al azar con tres tratamientos experimentales correspondientes a TC: testigo sin fertilización, TQ: aplicación de $60 \mathrm{~kg}$ de nitrógeno $\mathrm{ha}^{-1}$ y $40 \mathrm{~kg}$ de fósforo $\mathrm{ha}^{-1}$, empleando urea (50-00-00) y superfosfato triple (50-00-00) y TB: aplicación de la cepa T2 $\left(1 \times 10^{8} \mathrm{UFC} \mathrm{g}^{-1}\right)$.

Para el efecto de la aplicación de Trichoderma en campo, se empleó la cepa T2, en función de que el tiempo de obtención de conidios fue más rápida. Cada tratamiento consistió en tres repeticiones, para un total de 9 unidades experimentales, cada una con 10 plantas repartidas de 39 surcos de 100 m y $0.8 \mathrm{~m}$ de ancho. A los 45 días después de la siembra (DDS) se realizaron las aplicaciones 'in drench' de los tratamientos TQ y TB.

Dos días después de la aplicación se iniciaron mediciones semanales hasta los 108 DDS de la altura de planta, diámetro del tallo, número de flores y de vaina y al finalizar el ciclo se midió la longitud de la raíz, peso de la planta y rendimiento. La altura de la planta se midió a partir del cuello al ápice empleando un flexómetro; el diámetro se consideró de la parte media de la planta y se evaluó con un vernier digital. 
Posterior a la cosecha, se determinó la longitud de la raíz con un flexómetro; el rendimiento en grano se estimó obteniendo el peso de los granos de las plantas cosechadas por unidad experimental y transformada a rendimiento por hectárea a una humedad de $12 \%$. Los datos obtenidos se analizaron con el software SAS v. 9, empleando un análisis de varianza y comparación de medias Tukey $(p \leq 0.05)$.

\section{Resultados y discusión}

\section{Confrontaciones in vitro con Trichoderma harzianum (cultivos duales)}

De acuerdo con la escala descrita por Bell et al. (1982), el nivel de antagonismo de T1 frente a las cepas de Fusarium fue de clase 1 ya que la cepa T1 invadió la superficie de la caja. En tanto en las confrontaciones con S. rolfsii, M. phaseolina (M-Sonora) y (M-GTO) el nivel de antagonismo de T1 se encontró en clase 2, debido a que solo cubrió dos terceras partes del área de crecimiento de los patógenos (Figura 1).
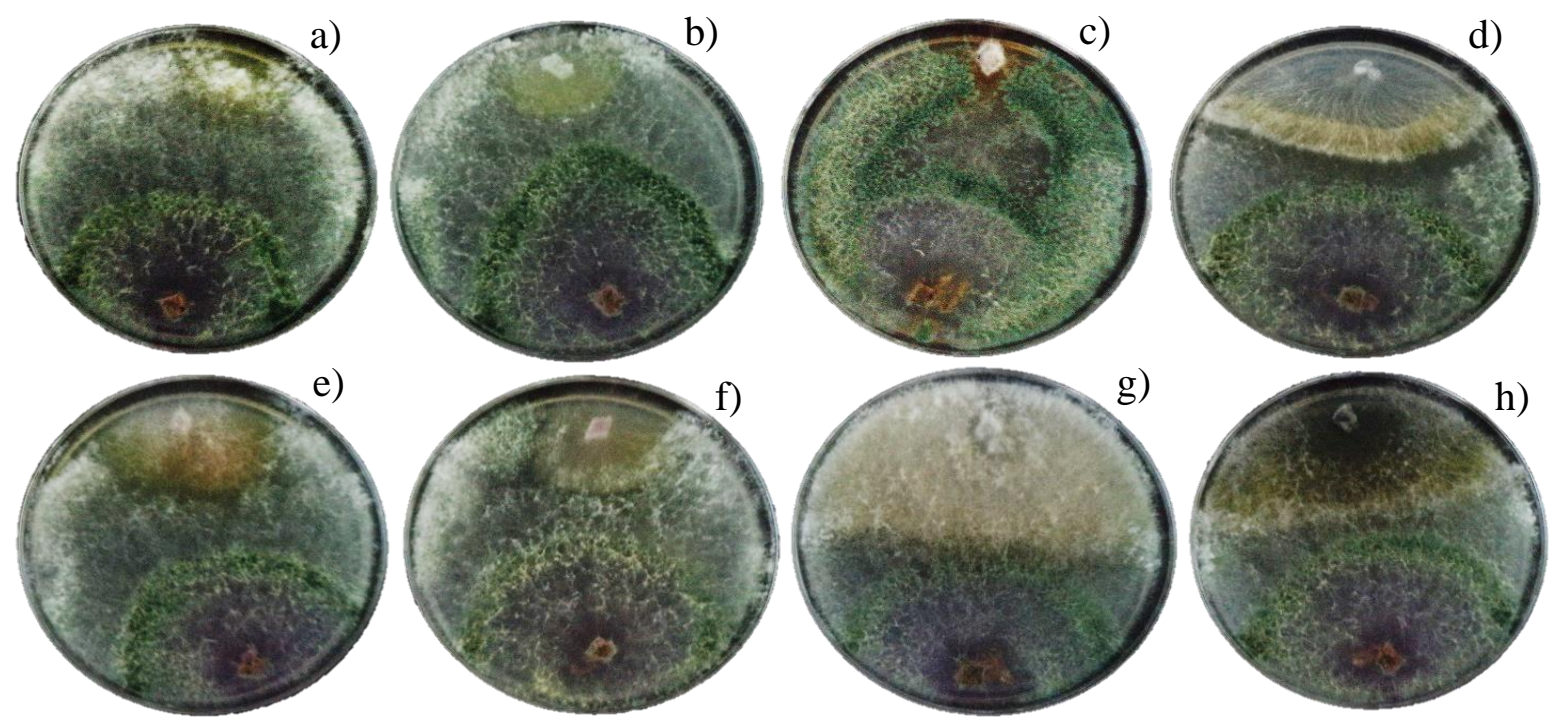

Figura 1. Evaluación antagónica in vitro cepa T1 (cultivos duales). Evaluación de la interacción 7 días de crecimiento. a) Foc 250, micoparasitismos y competencia por nutrientes; b) Foc 71, micoparasitismos y competencia por nutrientes; c) $F$. solani, micoparasitismos y posible antibiosis; d) $S$. rolfsii, micoparasitismos y competencia por nutrientes; e) Foc 27, micoparasitismos y presencia de enzimas antifungicas; f) Foc 12, micoparasitismos y competencia por nutrientes; y g) M-Sonora, micoparasitismos y competencia por nutrientes y H) M-GTO, micoparasitismos y competencia por nutrientes.

Según los criterios de Widyastuti (2006), la cepa T1 exhibió mayor crecimiento con respecto al crecimiento de los patógenos asociados a la fusariosis vascular del garbanzo. Frente a las cepas Foc 250, Foc 71, Foc 12 se presentó micoparasitismo leve y competencia por los nutrientes; con F. solani posible antibiosis, mientras que con Foc 27 hubo producción de enzimas antifúngicas debido al cambio de coloración rosa pálido en el sitio de contacto. 
En cuanto a $S$. rolfsii, presentó micoparasitismo moderado y competencia por nutrientes, las dos cepas de $M$. phaseolina (M-Sonora) y (M-GTO) mantuvieron micoparasitismo moderado y competencia por nutrientes. En cuanto a la cepa T2, se obtuvo un comportamiento similar de colonización de T1, el antagonismo contra las cepas Foc 250, Foc 27, Foc 71 y el Foc 12, F. solani, $S$. rolfsii y $M$. phaseolina (M-GTO) se encontró en la clase 1, mientras que con la cepa M-Sonora se observó una colonización de la mitad de la superficie del medio, lo que correspondió a un nivel de antagonismo clase 2 (Figura 2).
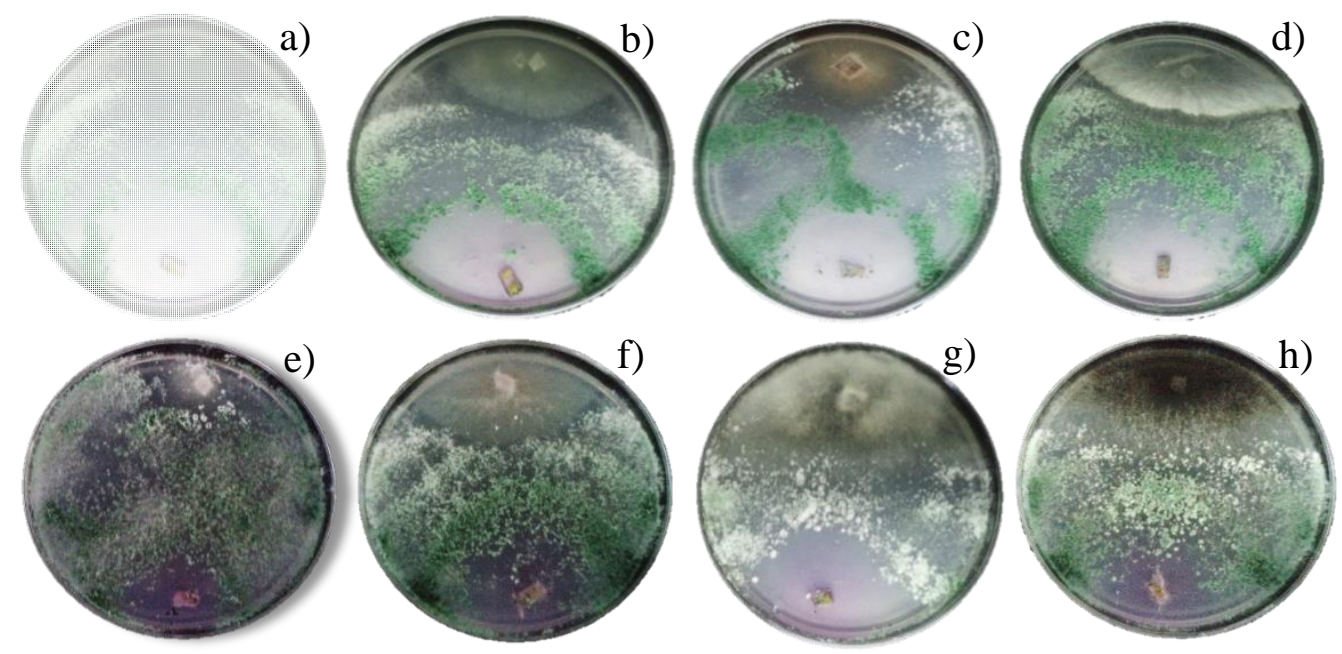

Figura 2. Evaluación antagónica in vitro cepa T2 (cultivos duales). Evaluación de la interacción 7 días de crecimiento. a) Foc 250, micoparasitismo y competencia por nutrientes; b) Foc 71, micoparasitismo y competencia por nutrientes; c) $F$. solani, micoparasitismo y competencia por nutrientes; d) $S$. rolfsii, micoparasitismo y competencia por nutrientes; e) Foc 27, micoparasitismo; f) Foc 12, micoparasitismo y competencia por nutrientes; g) M-Sonora, micoparasitismo y competencia por nutrientes; y h) M-GTO, micoparasitismo y competencia por nutrientes.

En cuanto al mecanismo de antagonismo, siete de las cepas del patógeno presentaron micoparasitismo y competencia por nutrientes, excepto la cepa Foc 27 que exhibió micoparasitismo y crecimiento rápido (Widyastuti, 2006) Otros estudios han coincidido que especies de Trichoderma mantienen valores de micoparasitismo frente a fitopatógenos en una escala de 1 (invasión completa); 2 (invasión a dos terceras partes); y 3 (Trichoderma y el fitopatógeno colonizan cada uno la mitad de la superficie) (Michel-Aceves et al., 2009).

Según Guédez et al. (2012) una característica principal en la elección de cepas de Trichoderma para emplearse en el control biológico es la agresividad para micoparasitar y la velocidad de crecimiento, esta debe superar a la del patógeno a controlar. La invasión del micelio que ejerció Trichoderma confirmó su efecto hiperparásitico que redujo el desarrollo de micelios de los patógenos, se han señalado diferentes mecanismos de antagonismo que van desde la competencia por nutrientes y espacio, producción de metabolitos antifúngicos y enzimas hidrolíticas.

Las propiedades antagónicas de este hongo se basan en la activación de múltiples mecanismos que también promueven la producción de compuestos específicos y metabolitos que funcionan como factores de crecimiento de plantas y mejoran su resistencia sistémica contra las enfermedades 
(Hernández-Melchor et al., 2019). A nivel de microscopio, los mecanismos de micoparasitismo de las cepas T1 y T2 sobre las ocho cepas fitopatógenas se observaron de forma general a través del enrollamiento, penetración y vacuolización (Figura 3).
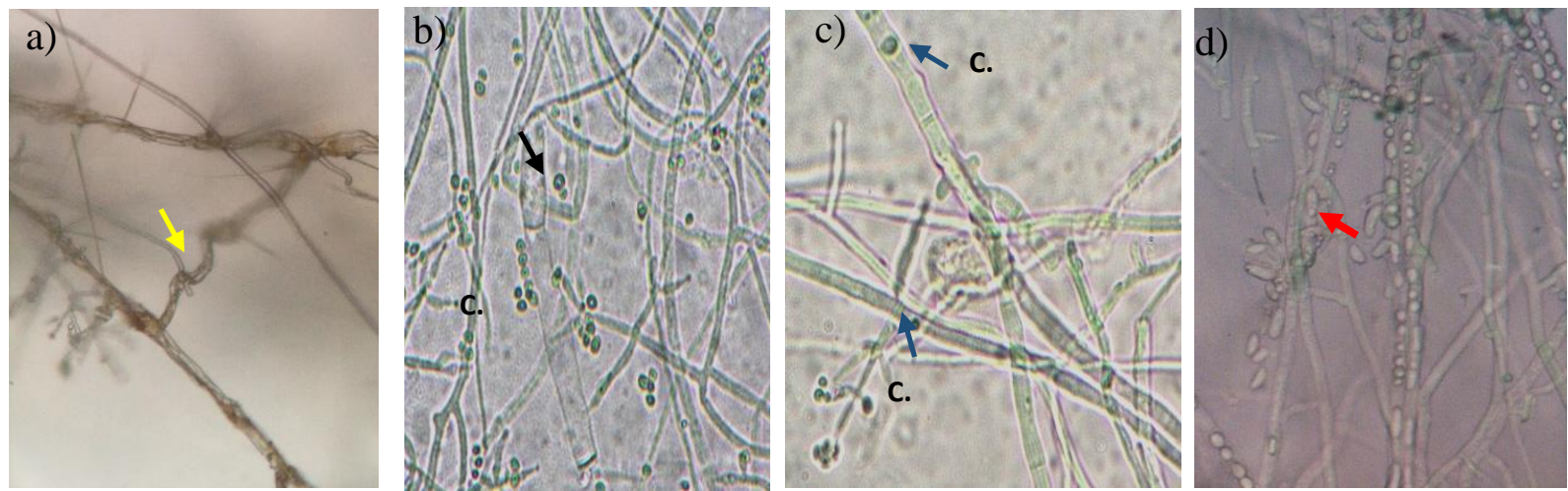

Figura 3. Mecanismos de micoparasitismo de T2 contra a Foc 250. a) enrollamiento (flecha amarilla); b) penetración (flecha negra); c) penetración flecha azul; y d) vacuolización (flecha roja).

Diferentes investigaciones indican que el proceso de micoparasitismo es el mejor mecanismo antagónico mostrado por Trichoderma, éste inicia cuando reconoce al hospedante y se une a las hifas mediante apresorios, posteriormente degrada la pared celular mediante la secreción de enzimas, principalmente quitinasas y $\beta$-1,3-glucanasas, celulasas, proteasas y fosfatasas (Qualhato et al., 2013).

Los valores de porcentaje de inhibición de crecimiento radial (PICR) en las ocho cepas evaluadas se encontraron en un intervalo de 49 a $85 \%$ (Cuadro 2). De acuerdo con el análisis de varianza ( $p \leq$ 0.05) se observó una diferencia significativa en los PICR con respecto a T1 y T2 y su efecto sobre los ocho patógena (Cuadro 2). La cepa T1 presentó mayor inhibición frente a $F$. solani, Foc 250, Foc 71 y Foc 12.

Cuadro 2. Porcentaje de inhibición del crecimiento radial (PICR) de los patógenos evaluados contra las cepas de Trichoderma harzianum T1 y T2.

\begin{tabular}{ccc}
\hline \multirow{2}{*}{ Patógeno } & \multicolumn{2}{c}{ PICR (\%) } \\
\cline { 2 - 3 } & T1 & T2 \\
\hline Foc 250 (raza 5) & $78.5 \mathrm{~b}$ & $78.3 \mathrm{a}$ \\
Foc 71(raza 0) & $72.6 \mathrm{bc}$ & $70.9 \mathrm{c}$ \\
Fusarium solani & $85.8 \mathrm{a}$ & $80.8 \mathrm{a}$ \\
Sclerotium rolfsii & $63.1 \mathrm{de}$ & $74.3 \mathrm{ba}$ \\
Foc 27 (raza 1B/C) & $67.5 \mathrm{~d}$ & $80 \mathrm{a}$ \\
Foc 12 (raza 6) & $72.9 \mathrm{bc}$ & $76.6 \mathrm{ba}$ \\
M-Sonora & $49 \mathrm{f}$ & $75.3 \mathrm{ba}$ \\
M-GTO & $60 \mathrm{de}$ & $65.3 \mathrm{~d}$ \\
\hline
\end{tabular}

Medias con la misma literal entre columnas, son estadísticamente iguales, Tukey $(p \leq 0.05)$. 
Un efecto similar se observó con T2 frente a F. solani, Foc 250 y Foc 71; sin embargo, la inhibición aumentó para Foc 27, S. rolfsii, M-GTO y M-Sonora, en este último el PICR fue considerable al observarse un aumento de 53\% con respecto a T1. Estos resultados coinciden con lo documentado por autores como Michel-Aceves et al. (2009) quienes indicaron PICR en un intervalo de 16.4 a $77.8 \%$ cuando evaluaron Trichoderma frente a $F$. oxysporum f. sp. lycopersici y 13.1 a $94.4 \%$ para S. rolfsii.

En otro estudio realizado por Rudresh et al. (2005) empleando T. harzianum, obtuvieron valores de inhibición en un rango de 12.2 a $59.9 \%$ para $S$. rolfsii y de 65.2 a $77 \%$ para $F$. oxysporum f. sp. ciceri. En tanto, para M. phaseolina Salazar et al. (2012) determinaron PICR en intervalos de 67 al 91\%; mientras que Cubilla-Ríos et al. (2019) obtuvieron inhibición entre 55.6 a 52.8\%.

\section{Efecto de $T$. harzianum en el cultivo de garbanzo}

Las plantas inoculadas con TB (aplicación de la cepa de Trichoderma T2) presentaron incrementos en altura de $2.2,3$ y $2.3 \%$ en los días 66,73 y 81 , respectivamente, comparadas con el control (Figura 4a); mientras que con TQ (aplicación de fertilizante químico) los valores fueron 1.3\% menores. Con respecto al diámetro del tallo, las principales diferencias se observaron a partir del día 94, durante este periodo las plantas con la aplicación de Trichoderma y la fertilización química presentaron incrementos de 3.3 y $3.5 \%$, respectivamente (Figura $4 b$ ).
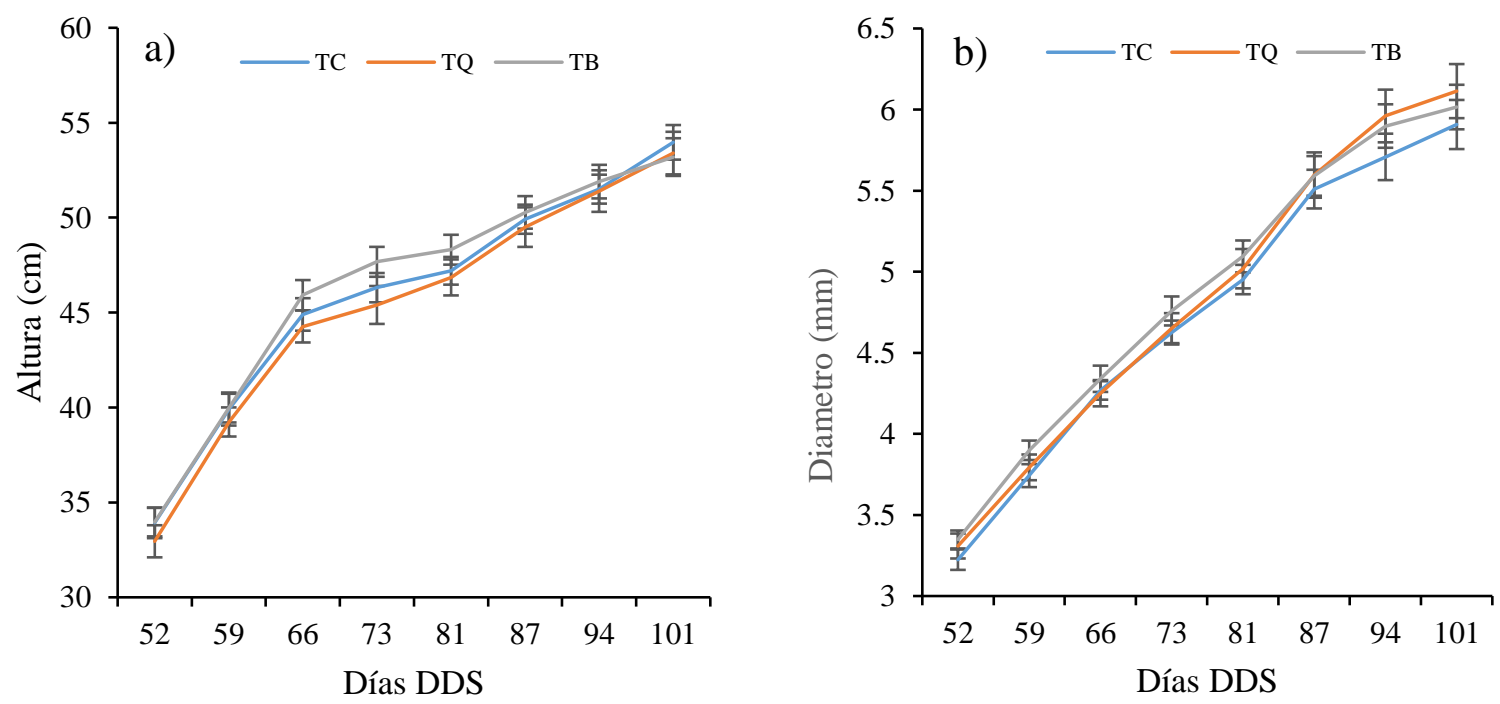

Figura 4. Evaluación de la altura (a) y diámetro del tallo (b) en plantas de garbanzo. $\mathbf{n = 3 0}$.

El efecto en la altura y diámetro de las plantas que se encontraron en este estudio fueron menores en comparación con otros estudios, como el documentado por Boureghda y Bouznad (2009), quienes aplicaron $T$. harzianum en plantas de garbanzo, sus resultados indicaron aumentos en promedio de $8 \%$ en la altura de las plantas. En tanto Oliva-Ortíz et al. (2017) al evaluar el efecto de cepas de Trichoderma obtuvieron incrementos de $26 \%$ con respecto al testigo, adicionalmente, observaron un incremento del diámetro del tallo del $8 \%$ con respecto al control. 
En relación con la variable número de flores y de vainas, se observó que TB estimuló la floración a partir del día 56, de esta forma, para el día 66 se estimó un aumento de $30 \%$ con respecto al control, mientras que con el tratamiento TQ se determinó 10\% menos de flores con relación al control. En general, no se observaron diferencias significativas $(p<0.05)$. Después del día 81 ambos tratamientos presentaron un comportamiento similar (Figura 5a).

En cuanto a la formación de vainas con TB hubo un aumento de estas a partir del día 73 con respecto al control, mientras que con TQ esta tuvo un ligero aumento a partir del día 87, en promedio el aumento fue de 24 y $11 \%$, con TB y TQ, respectivamente (Figura 5b). Autores como Mukherjee et al. (2019) encontraron adelanto de la floración (7-10 días) en garbanzo y lenteja cuando aplicaron $T$. virens, además de observar $26 \%$ de incremento en la producción.

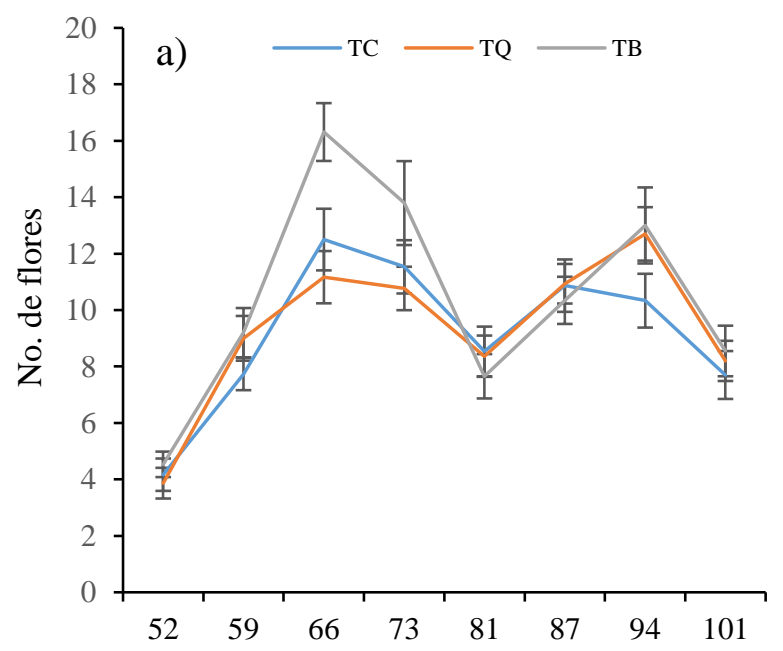

Días DDS

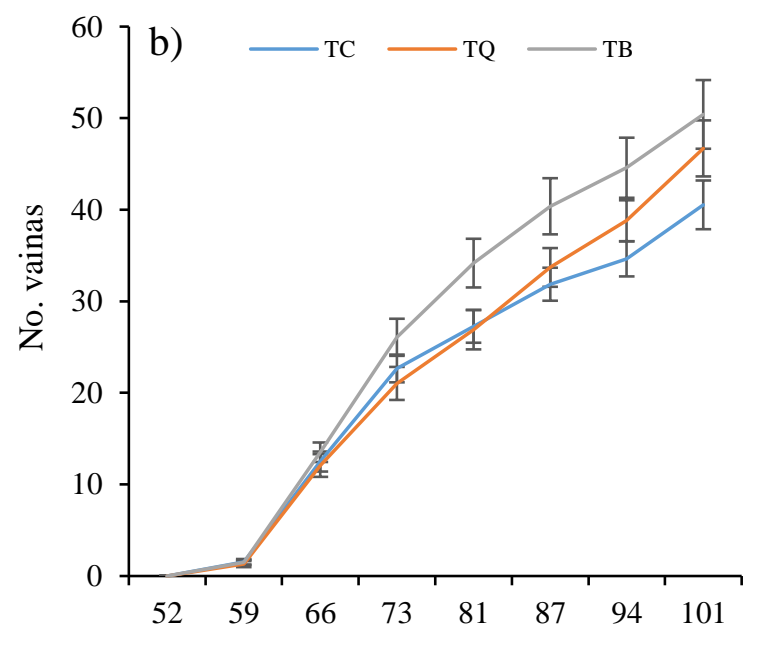

Días DDS

Figura 5. Evaluación del número de flores (a) y vainas (b) en plantas de garbanzo. $\mathbf{n}=\mathbf{3 0}$.

En este sentido Mishra y Nautiyal (2018) obtuvieron incrementos de $20 \%$ en la producción de vainas de garbanzo cuando aplicaron $T$. viride. En otro estudio realizado por Ávila-Miramontes $e t$ al. (2015) donde evaluaron diferentes tratamientos a base de T. harzianum, B. subtilis, Mesorhizobium ciceri y fertilización nitrogenada, pudieron observar que con la combinación $T$. harzianum + fertilización se tuvo mayor precocidad y emisión constante de flores, así como un periodo largo de fructificaciones.

Para la variable longitud de raíz se determinó diferencia significativa $(p \leq 0.05)$ entre tratamientos TB y TQ causaron un aumento de 40 y $13 \%$, respectivamente, con referencia al control (Cuadro 3). Este comportamiento se ha presentado en diversos estudios en los que se aplica Trichoderma y que registran incrementos en la longitud de la raíz, debido a la estimulación de la producción de hormonas, principalmente auxinas, las que son sintetizadas a partir de fuente de triptófano que se secretan naturalmente en los exudados de las raíces de las plantas (Hoyos-Carvajal et al., 2009). 
Cuadro 3. Variables agronómicas determinadas después de la cosecha.

\begin{tabular}{ccc}
\hline Tratamientos & Longitud de raíz $(\mathrm{cm})$ & ${\text { Rendimiento }\left(\mathrm{t} \mathrm{ha}^{-1}\right)}^{-10.3 \mathrm{~b}}$ \\
\hline Control (TC) & $11.7 \mathrm{~b}$ & $2.1 \mathrm{a}$ \\
Químico (TQ) & $14.4 \mathrm{a}$ & $2.4 \mathrm{a}$ \\
Biológico (TB) & 2.7 & $2.6 \mathrm{a}$ \\
DMS & 1.7 \\
\hline
\end{tabular}

Medias con la misma literal entre columnas, son estadísticamente iguales, Tukey $(p \leq 0.05)$.

Es entonces, que el incremento de la raíz permite a las plantas abarcar volúmenes extensos de suelo y aumentar la absorción de nutrientes disponibles y los que han sido solubilizados por el mismo hongo (Jyotsna et al. 2008; Hoyos-Carvajal et al., 2009). Al respecto Kumar et al. (2014) obtuvieron resultados similares al emplear $5 \%$ de $T$. harzianum con relación a un kg de semilla de garbanzo, adicionalmente, redujo la incidencia de la marchitez asociada al complejo $F$. oxysporum f. sp. ciceris, M. phaseolina y $S$. rolfsii.

De la misma forma, Jyotsna et al. (2008) detectaron incrementos en el tamaño de la raíz entre 25 a $27 \%$ y la disminución de enfermedades en 40 a $60 \%$, los autores indicaron que la estimulación de la producción de hormonas en etapas tempranas de crecimiento puede ayudar al crecimiento de la planta y el desarrollo de raíces. Hoyos-Carvajal et al. (2009) recomienda que cepas de Trichoderma promotoras de crecimiento pueden ser empleadas para la formulación de nuevos productos que sean benéficos para en la agricultura.

Con respecto al rendimiento, se determinó que con TQ hubo un aumento de $13 \%$ y con TB de $23 \%$, ambos datos con respecto a TC. Estos resultados coinciden con diferentes autores que señalan incrementos en el rendimiento de cultivos (Oliva-Ortiz et al., 2017; Ávila-Miramontes et al., 2015; Ruiz-Cisneros et al., 2018).

En garbanzo los estudios realizados con Trichoderma están enfocados al control de patógenos, principalmente, F. oxysporum f. sp. ciceris y de forma secundaría estiman los efectos en el crecimiento y producción de las plantas, de tal forma, que sus resultados indican la disminución de la severidad de la enfermedad, así como incrementos en el rendimiento, tal es el caso de OlivaOrtiz et al. (2017) quienes encontraron un aumento de más de 70\% al aplicar la cepa HRG-060.

Así como la disminución de incidencia y marchitez de raíz, los autores obtuvieron diferentes resultados entre cepas, señalando que no todas mantienen los mismos efectos con respecto a las variables estudiadas. En tanto, Khan et al. (2014) demostró que al hacer aplicaciones de Trichoderma en suelo obtuvieron reducción de la severidad de la enfermedad de $25 \%$ a $67 \%$, a la vez que obtuvieron incrementos de rendimientos en un intervalo de 8 a 24\%. Así también, Kumar et al. (2014) demostró que la aplicación de T. harzianum incrementó el rendimiento en $68 \%$ y al mismo tiempo la incidencia disminuyó en $91 \%$.

Los incrementos en producción pueden atribuirse por un lado a la protección antagónica de Trichoderma sp. Contra agentes causales de enfermedades en la planta y por otro lado a la síntesis de compuestos reguladores de crecimiento o que intervienen en la asimilación de nutrientes para la planta (Hoyos-Carbajal et al., 2009; Hernández-Melchor et al., 2019). 
Asimismo, en algunos cultivos como fríjol se reconoce que Trichoderma sp. Favorece la germinación de la semilla a través de la producción de enzimas líticas que degradan el epispermo, el cual es una cubierta seminal que constituye a la testa; adicionalmente, el hongo es capaz de promover el desarrollo de los tejidos meristemáticos primarios, los que son responsables del crecimiento de la planta con relación a la altura, peso y desarrollo de raíces (Zúñiga-Silgado y Vélez-Vargas, 2016).

De acuerdo a los resultados que se obtuvieron con $\mathrm{T} 1$ y $\mathrm{T} 2$, ambas cepas tuvieron potencial para usarse en el control de patógenos, así como para el incremento de las características agronómicas que se evaluaron; sin embargo, considerando las experiencia de los productores es necesario realizar evaluaciones para determinar la dosis óptima de aplicación Trichoderma (UFC $\mathrm{g}^{-1}$ ) considerando en las condiciones de cada unidad de producción, principalmente el nivel de inóculo inicial de fitopatógenos, la disponibilidad de nutrientes y las condiciones climáticas y edafológicas del área.

\section{Conclusiones}

De acuerdo con los resultados de esta investigación ambas cepas de T. harzianum (T1 y T2) presentaron potencial para emplearse en el control de la fusariosis vascular en el cultivo del garbanzo. Los mecanismos de antagonismos que exhibieron ambas cepas fue la competencia por nutrientes, micoparasitismos, producción de enzimas antifungicas y posible antibiosis. La cepa T1 presentó mayor inhibición frente a las cepas FOC 250, FOC 71 y Fusarium solani, mientras que T2 para Sclerotium rolfsii, FOC 27, FOC 12 y para los aislados de Macrophomina phaseolina. Adicionalmente, la aplicación de Trichoderma permitió incrementos en la producción de vainas (24\%), longitud de la raíz (40\%) y rendimiento (23\%).

\section{Agradecimientos}

El presente trabajo fue desarrollado con recursos otorgados a través de proyectos fiscales-2013 del INIFAP mediante el convenio Núm. 15534132023.

\section{Literatura citada}

Abdel-Monaim, M. F.; Abo-Elyousr, K. A. M. and Morsy, K. M. 2011. Effectiveness of plant extracts on suppression of damping-off and wilt diseases of lupine (Lupinus termis Forsik). Crop Prot. 30(2):185-191.

Arvayo-Ortiz, R. M.; Esqueda, M.; Acedo-Felix, E.; Sánchez, A. and Gutiérrez, A. 2011. Morphological variability and races of Fusarium oxysporum f. sp. ciceris associated with chickpea (Cicer arietinum) crops. Am. J. Agric. Biol. Sci. 6(1):114-121.

Ávila-Miramontes, J. A.; Padilla-Zaldo, G.; Martínez-Heredia, D.; Rivas-Santoyo, F. J.; CoronadoEspericueta, M. A. y Ortega-Murrieta, P. 2015. Respuesta de algunos componentes del rendimiento del cultivo de garbanzo (Cicer arietinum 1.) a la inoculación de Mesorhizobium ciceri, Trichoderma harzianum y Bacillus subtilis en la región agrícola de la costa de Hermosillo. Biotecnia. 17(3):3-8.

Bell, D. K.; Wells, H. D. and Markam, C. R. 1982. In vitro antagonism of Trichoderma spp. against six fungal pathogens. Phytopathol. 72(4):379-382. 
Boureghda, H. and Bouznad, Z. 2009. Biological control of Fusarium Wilt of chickpea using isolates of Trichoderma atroviride, $T$. harzianum and T. longibrachiatum. Acta Phytopathol. Entomol. Hung. 44(1):25-38.

Cubilla-Rios, A. A.; Ruíz-Díaz-Mendoza, D. D.; Romero-Rodríguez, M. C.; Flores-Giubi, M. E. y Barúa-Chamorro, J. E. 2019. Antibiosis de proteínas y metabolitos en especies de Trichoderma contra aislamientos paraguayos de Macrophomina phaseolina. Agron. Mesoam. 30(1):63-77.

Dennis, C. and Webster, J. 1971. Antagonistic properties of species-groups of Trichoderma: II. Production of volatile antibiotics. Trans. Brit. Mycol. Soc. 57(1):41-47.

Zúñiga-Silgado, D. and Vélez-Vargas L. D. 2016. Evaluation of phosphodissolvent IAA producing strains of Trichoderma spp. through biometric response of Phaseolus vulgaris L. Int. J. Biosci. 8(6):103-118.

Guédez, C.; Cañizalez, L.; Castillo, C. y Olivar, R. 2012. Evaluación in vitro de aislamientos de Trichoderma harzianum para el control de Rhizoctonia solani, Sclerotium rolfsii y Fusarium oxysporum en plantas de tomate. Rev. Soc. Ven. Microbiol. 32(1):44-49.

Haware, M. P. and Nene, Y. L. 1982. Races of Fusarium oxysporum f. sp. ciceri. Plant Dis. 66(9):809-810.

Haware, M. P.; Nene, Y. L. and Natarajan, M. 1996. The survival of Fusarium oxysporum f. sp. ciceri in the soil in the absence of chickpea. Phytopathol. Mediterr. 35(1):9-12.

Hernández-Melchor, D. J.; Ferrera-Cerrato, R. y Alarcón, A. 2019. Trichoderma: importancia agrícola, biotecnológica, y sistemas de fermentación para producir biomasa y enzimas de interés industrial. Chil. J. Agric. Anim. Sci. 35(1):98-112.

Hoyos-Carvajal, L.; Orduz, S. and Bisset, J. 2009. Growth stimulation in bean (Phaseolus vulgaris L.) by Trichoderma. Biol. Control. 51(3):409-416.

Jiménez-Díaz, R. M.; Alcalá-Jiménez, A. R.; Hervás, A. and Trapero-Casas, J. L. 1993. Pathogenic variability and host resistance in the Fusarium oxysporum f. sp. ciceris/Cicer arietinum pathosystem. In: proceeding of the $3^{\text {rd }}$ European seminars Fusarium mycotoxins, taxonomy, pathogenicity and host resistance. Jiménez-Díaz, R. M.; Alcalá-Jiménez, A. R.; Hervás, A. and Trapero-Casas, J. L. (Comps.). Radzikóv, Poland. 87-94 pp.

Jiménez-Gasco, M. M.; Navas-Cortés, J. A. and Jiménez-Díaz, R. M. 2004. The Fusarium oxysporum f.sp. ciceris/ Cicer arietinum pathosystem: a case study of the evolution of plant-pathogenic fungi into races and pathotypes. Int. Microbiol. 7(2):95-104.

Jyotsna, A. S.; Rajat, P. S.; Alok, K. S.; Anil, K. S. and Dilip, K. A. 2008. Growth promotion and charcoal rot management in Chickpea by T. harzianum. J. Plant. Prot. Res. 48(1):81-92.

Khan, M. R.; Ashraf, S.; Rassol, F.; Salati, K. M.; Mohiddin, F. A. and Haque, Z. 2014. Field performance of Trichoderma species against wilt disease complex of chickpea caused by F. oxysporum f. sp. ciceri and Rhizoctonia solani. Turk. J. Agric. For. 38(4):447-454.

Khan, A. N.; Shair, F; Malik, K.; Hayat, Z.; Khan, M. A.; Hafeez, F. Y. and Hassan, M. N. 2017. Molecular identification and genetic characterization of Macrophomina phaseolina strains causing pathogenicity on sunflower and chickpea. Front Microbiol. 8:1309.

Kumar, V; Shahid, M.; Srivastava, M.; Singh, A.; Pandey, S. and Sharma. 2014. Enhancing seed germination and vigor of chickpea by using potential and effective Strains of Trichoderma Species. Virol. Mycol. 3(2):1-7.

Michel-Aceves, A. C.; Otero-Sánchez, M. and Solano-Pascacio, L. 2009. Biocontrol in vitro con Trichoderma spp. de Fusarium subglutinans (Wollenweb y Reinking) Nelson, Toussoun y Marasas y $F$. oxysporum Schlecht., agentes causales de la 'escoba de bruja' del mango (Mangifera indica L.). Rev. Mex- Fitopatol. 27(1):18-26. 
Mishra, A. and Nautiyal, C. S. 2018. Novel Trichoderma fusant for enhancing nutritional value and defence activity in chickpea. Physiol. Mol. Biol. Plants. 24(3):411-422.

Mukherjee, P. K.; Mehetre, S. T.; Sherkhane, P. D.; Muthukathan, G.; Ghosh, A.; Kotasthane, A. S.; Khare, N.; Rathod, P.; Sharma, K. K.; Nath, R.; Tewari, A. K.; Bhattacharyya, S.; Arya, M.; Pathak D.; Wasnikar, A. R.; Tiwari, R. K. S. and Saxena, D. R. 2019. A novel seeddressing formulation based on an improved mutant strain of Trichoderma virens, and its field evaluation. Front. Microbiol. 10:1910

Navas-Cortés, J. A.; Hau, B. and Jiménez-Díaz, R. M. 2000. Yield loss in chickpeas in relation to development of Fusarium wilt epidemics. Phytopathology. 90(11):1269-1278.

Oliva-Ortiz, L. C.; Velázquez-Alcaraz, T. J.; Sosa-Pérez, R.; Partida-Ruvalcaba, L.; Díaz-Valdés, T.; Arciniega-Ramos, J y López-Orona, C. A. 2017. Control de la fusariosis vascular del garbanzo (Cicer arietinum L.) por microorganismos nativos de Sinaloa, México. Agrociencia. 51(6):683-695.

Qualhato, T.; Cardoso-Lopes, F. A.; Steindorff, A. S.; Brandão, R. S.; Jesuino, R. S. A. and Ulhoa, C. J. 2013. Mycoparasitism studies of Trichoderma species against three phytopathogenic fungi: evaluation of antagonism and hydrolytic enzyme production. Biotechnol. Lett. 35(9):1461-1468.

Rajput, V. A.; Konde, S. A. and Thakur, M. R. 2010. Evaluation of bioagents against chickpea wilt complex. J. Soils Crops. 20(1):155-158.

Rudresh, D. I.; Shivaprakash, M. K. and Ravulapalli, D. 2005. Potential of Trichoderma spp. as biocontrol agents of pathogens involved in wilt complex of chickpea (Cicer arietinum L.). Biol. Control. 19(2):157-166.

Ruiz-Cisneros, M. F.; Ornelas-Paz, J. J.; Olivas-Orozco, G. I.; Acosta-Muñiz, C. H.; SepúlvedaAhumada, D. R.; Pérez-Corral, D. A.; Ríos-Velasco, C.; Salas-Marina, M. A. and Fernández-Pavía, S. P. 2018. Efecto de Trichoderma spp. y hongos fitopatógenos sobre el crecimiento vegetal y calidad del fruto de jitomate. Rev. Mex. Fitopatol. 36(3):444-456.

Salazar, L. A.; Aponte, G. Y.; Alcano, M. J.; Sanabria, N. H. y Guzmán, J. J. 2012. Importancia de las especies de Trichoderma para el control de Macrophomina phaseolina en las áreas agrícolas del estado Aragua, Venezuela. Agron. Trop. 62(1-4):7-15.

Sharma, M. and Ghosh, R. 2017. Heat and soil moisture stress differentially impact chickpea plant infection with fungal pathogens. In: plant tolerance to individual and concurrent stresses. Senthil-Kumar M. (Ed.). Springer press. New Delhi. 47-57 pp.

Suárez, C. L.; Fernández, R. J.; Valero, N. O.; Gomez, R. M. y Paez, A. R. 2008. Antagonismo in vitro de Trichoderma harzianum Rifai sobre Fusarium solani (Mart.) Sacc., asociado a la marchitez en maracuyá. Rev. Colomb. Biotecnol. 10(2):35-43.

Widyastuti, S. M. 2006. The biological control of Ganoderma root rot by Trichoderma. Heart rot and root rot in tropical Acacia plantations. In: Potter, K.; Rimbawanto, A. and Beadle, C. (Eds.). Proceedings of a workshop held in Yogyakarta, Indonesia. 7-9 February 2006. Canberra. ACIAR Proceedings 124. 67-74 pp. 